Review Article

\title{
Effectiveness of Acupuncture on Urinary Retention: A Meta-Analysis
}

\author{
Chengwen Zheng, ${ }^{1}$ Zaoying $\mathrm{Li}^{2}{ }^{2}$ Haizhen $\mathrm{Lu} \mathbb{D}^{1},{ }^{1}$ and $\mathrm{Yi}$ Zhou $\mathbb{D}^{1}$ \\ ${ }^{1}$ Basic Medical School of Chengdu University of Traditional Chinese Medicine, Chengdu 610000, China \\ ${ }^{2}$ Clinical Medical School of Chengdu University of Chinese Medicine, Chengdu 610000, China \\ Correspondence should be addressed to Yi Zhou; zhoumen1123456@163.com
}

Received 17 June 2021; Accepted 14 September 2021; Published 29 September 2021

Academic Editor: Jih-Huah Wu

Copyright (c) 2021 Chengwen Zheng et al. This is an open access article distributed under the Creative Commons Attribution License, which permits unrestricted use, distribution, and reproduction in any medium, provided the original work is properly cited.

\begin{abstract}
Objectives. This study aimed to evaluate the safety and efficacy of acupuncture in the treatment of urinary retention (UR). Methods. Randomized controlled trials investigating the effectiveness of acupuncture in the treatment of UR were identified by searching seven comprehensive databases (Cochrane Library, PubMed, Embase, China National Knowledge Infrastructure, Wanfang Database, China Science and Technology Journal Database, and Chinese Biomedical Literature Database) prior to September 2020. Data analysis was performed using RevMan, version 5.3, and Stata software, version 14.0. Results. A total of 12 studies with 979 participants were included. A random-effects model was used to conduct a meta-analysis on the acupuncture group and the control group. The results show that acupuncture can effectively promote spontaneous urination and reduce anxiety in patients with poor urination (relative risk: $1.35 ; 95 \%$ confidence interval $(\mathrm{CI}): 1.19-1.53 ; P<0.00001)$. The random-effects model showed significant differences in residual urine volume between the acupuncture group and the control group (MD: $-84.79,95 \% \mathrm{CI}$ : -135.62 to $-33.94 ; P=0.001)$. Conclusion. Acupuncture is safe and effective in the treatment of UR. However, since the current level of evidence is limited, high-quality, large-sample, multi-center, clinical randomized controlled trials are needed to further confirm our conclusions in the future.
\end{abstract}

\section{Introduction}

Urinary retention (UR), which is characterized by an inability to completely empty the bladder, is a common complication of surgery and anesthesia [1]. However, there is currently no standardized definition of UR. It is generally believed that a remaining urinary volume of $>300 \mathrm{ml}$ in the bladder is the diagnostic basis for UR $[2,3]$. The incidence of UR is between $5 \%$ and $70 \%$, and the risk of UR increases with age, which seriously affects quality of life for patients $[4,5]$. However, there are currently no definitive and effective treatments for UR [6-8]. Although bladder function training, physical therapy, surgical treatment, drug treatment, and other methods are widely used in the clinic [9-13], one trial has shown that bladder training by catheter clamping offers no advantage over free-drainage removal of short-term urinary catheters [14]. Catheterization is generally considered the best treatment option for UR, but it increases the risk of urinary tract infection [15]. Although cholinergic drugs and prostaglandins are promising treatments for UR, there is still insufficient evidence to support their use [16].

Acupuncture, which encompasses a wide range of treatments, is recommended in various clinical guidelines for the treatment of multiple diseases, such as dysmenorrhea, rhinitis, and stroke [17-19]. At present, a large number of studies have shown that acupuncture regulates neurotransmitters and can objectively improve urodynamics [20, 21]. However, the European Association of Urology and the American Urological Association have not yet included acupuncture in treatment guidelines $[22,23]$. Therefore, we conducted a meta-analysis and merged data to provide clinical decision-making recommendations. In this metaanalysis, we identified clinical randomized controlled trials 
on acupuncture and moxibustion for UR and distinguished the commonly used acupuncture point selection schemes to clarify the curative effect of acupuncture on UR.

\section{Methods}

The systematic review protocol was developed using guidance from the Preferred Reporting Items for Systematic Reviews and Meta-Analyses (PRISMA) statement and is registered in PROSPERO (CRD42021228237).

2.1. Search Strategy. Randomized controlled trials were retrieved by searching the following databases from the date of inception to September 2020: Cochrane Library, PubMed, Embase, China National Knowledge Infrastructure, Wanfang Database, China Science and Technology Journal Database, and Chinese Biomedical Literature Database. No language restrictions were applied. According to the characteristics of each database, the search terms were as follows: ("acupuncture" OR "electro-acupuncture" OR "acupuncture points" OR “moxibustion”) AND (“urinary retention” OR "post-operative complications" OR "urination disorders"). Table 1 shows the PubMed search strategy as an example.

This search strategy was modified as required for other electronic databases.

\subsection{Inclusion and Exclusion Criteria}

2.2.1. Study Types. Only randomized controlled trials were included, regardless of blinding. Studies were written in Chinese or English.

2.2.2. Participants. Participants were patients with clinically confirmed UR, regardless of type, sex, age, and country.

2.2.3. Intervention. The experimental group underwent several types of acupuncture therapy, including electric, auricular, manual, and scalp acupuncture. Moxibustion, warm needle, fire needle, and acupoint injections were excluded. The control group received no intervention, placebo, drug therapy, rehabilitation training, sham acupuncture, or other conservative treatment, such as urinary catheterization. Studies comparing acupuncture plus traditional Chinese medicine with traditional Chinese medicine, as well as those comparing acupuncture plus moxibustion with moxibustion, were excluded.

2.2.4. Outcomes. The main outcome indicators were the change in the number of voluntary urinations and the change in residual urine volume (RUV).

2.3. Study Selection. All studies were imported into NoteExpress, and two reviewers (Chengwen Zheng and Zaoying $\mathrm{Li}$ ) independently screened them. After reading the titles and abstracts, duplicate articles and articles that did not meet the inclusion criteria were excluded. Studies that met the aforementioned predetermined inclusion criteria were included. Any inconsistency in the data extracted was resolved by a third reviewer (Haizhen Lu). A PRISMA flow diagram was used to describe the process of study selection.

2.4. Data Extraction. Data extraction was performed independently by two reviewers (Chengwen Zheng and Zaoying $\mathrm{Li})$. After comparing the results and verifying the original documents, the authenticity and completeness of all data were finally determined. The extracted content included author, publication year, sample size, male to female ratio, age of subjects, method used in the study, intervention measures used in each group, evaluation times of outcome indicators, evaluated outcome indicators, and each acupuncture point selected by the research institute. All disagreements were resolved by consensus between the two initial reviewers and the third reviewer (Haizhen $\mathrm{Lu}$ ).

2.5. Risk of Bias in Individual Studies. Two reviewers (Chengwen Zheng and Zaoying Li) assessed the risk of bias in included studies using the Cochrane Collaboration's riskof-bias tool. Each study was classified as having low, unclean, or high-risk bias based on seven items: (1) random sequence generation; (2) allocation concealment; (3) blinding of participants and personnel; (4) blinding of outcome assessment; (5) incomplete outcome data; (6) selective reporting; and (7) other. Any disagreements were resolved by the third reviewer (Haizhen Lu). The risk-of-bias assessment was summarized using Review Manager 5.3.

2.6. Data Analysis. A quantitative analysis was performed using Cochrane Collaboration software (RevMan, version 5.3.5) for the meta-analysis and using Stata software version 14.0 for funnel plot analysis. The relative risk (RR) with $95 \%$ confidence interval (CI) was selected as the statistic for dichotomous data. Because the unit of the outcome indicator is consistent, the mean difference (MD) with $95 \% \mathrm{CI}$ is used to describe continuous variables. The random-effects model was used to analyze all data. During the heterogeneity test, the chi-square test was performed first, and based on its findings, estimates of heterogeneity $\left(?^{2}\right)$ were applied. If heterogeneity was high, the source of heterogeneity was explored. A subgroup or sensitivity analysis was performed to investigate the stability of the meta-analysis. Publication bias was explored using a funnel plot analysis.

\section{Results}

3.1. Study Selection. A total of 4,180 unique studies were identified using the search strategy. After duplicate studies were excluded, 3,014 related studies were screened out. After reading the titles and abstracts of these studies, 17 related studies were identified. Further screening for eligibility was performed by two independent reviewers according to the inclusion and exclusion criteria. Eventually, 12 studies were included in the meta-analysis (Figure 1). 
Table 1: PubMed search strategy.

\begin{tabular}{|c|c|}
\hline Number & Search terms \\
\hline$\# 1$ & “Acupuncture"[Mesh] \\
\hline$\# 2$ & Acupuncture Therapy[Title/Abstract] \\
\hline \#3 & acupressure[Title/Abstract] \\
\hline$\# 4$ & Electroacupuncture* [Title/Abstract] \\
\hline \#5 & electro-acupuncture ${ }^{*}[$ Title/Abstract] \\
\hline \#6 & acupoint ${ }^{*}[$ Title/Abstract $]$ \\
\hline \#7 & meridian* ${ }^{*}$ Title/Abstract] \\
\hline$\# 8$ & non-meridian* [Title/Abstract] \\
\hline$\# 9$ & trigger* [Title/Abstract] \\
\hline$\# 10$ & Moxibustion [Title/Abstract] \\
\hline$\# 11$ & moxa $^{*}[$ Title/Abstract $]$ \\
\hline$\# 12$ & Acupuncture points[Title/Abstract] \\
\hline \#13 & auriculotherapy [Title/Abstract] \\
\hline & zhenjiu[Title/Abstract] OR zhen jiu [Title/Abstract] OR zhenci[Title/Abstract] OR zhen ci[Title/Abstract] OR cizhen[Title/ \\
\hline \#14 & $\begin{array}{l}\text { Abstract] OR dianzhen[Title/Abstract] OR dian zhen[Title/Abstract] OR zhen ya[Title/Abstract] OR er zhen[Title/Abstract] OR } \\
\text { ti zhen[Title/Abstract] OR she zhen[Title/Abstract] OR tou pi zhen[Title/Abstract] OR xue wei[Title/Abstract] }\end{array}$ \\
\hline \#15 & $\begin{array}{c}(((\text { ching}[\text { Title/Abstract] }) \text { AND (lo[Title/Abstract])) OR (jing[Title/Abstract] AND Luo[Title/Abstract])) OR (jinglo[Title/ } \\
\text { Abstract]) }\end{array}$ \\
\hline \#16 & $\# 1$ or $\# 2$ or $\# 3$ or $\# 4$ or $\# 5$ or $\# 6$ or $\# 7$ or $\# 8$ or $\# 9$ or $\# 10$ or $\# 11$ or $\# 12$ or $\# 13$ or $\# 14$ or $\# 15$ \\
\hline \#17 & Urinary Retention[MeSH terms] \\
\hline \#18 & post-operative Complications[Title/Abstract] \\
\hline \#19 & Urination Disorders[Title/Abstract] \\
\hline \#20 & (urin* ${ }^{*}$ Title/Abstract] AND (retent*[Title/Abstract] OR retain* $[$ Title/Abstract] $\left.)\right)$ \\
\hline \#21 & ((bladder* [Title/Abstract] OR void*[Title/Abstract]) AND (retent*[Title/Abstract] OR retain*[Title/Abstract])) \\
\hline$\# 22$ & $\# 17$ or \#18 or \#19 or \#20 or \#21 \\
\hline \#23 & $\# 16$ and \#22 \\
\hline
\end{tabular}

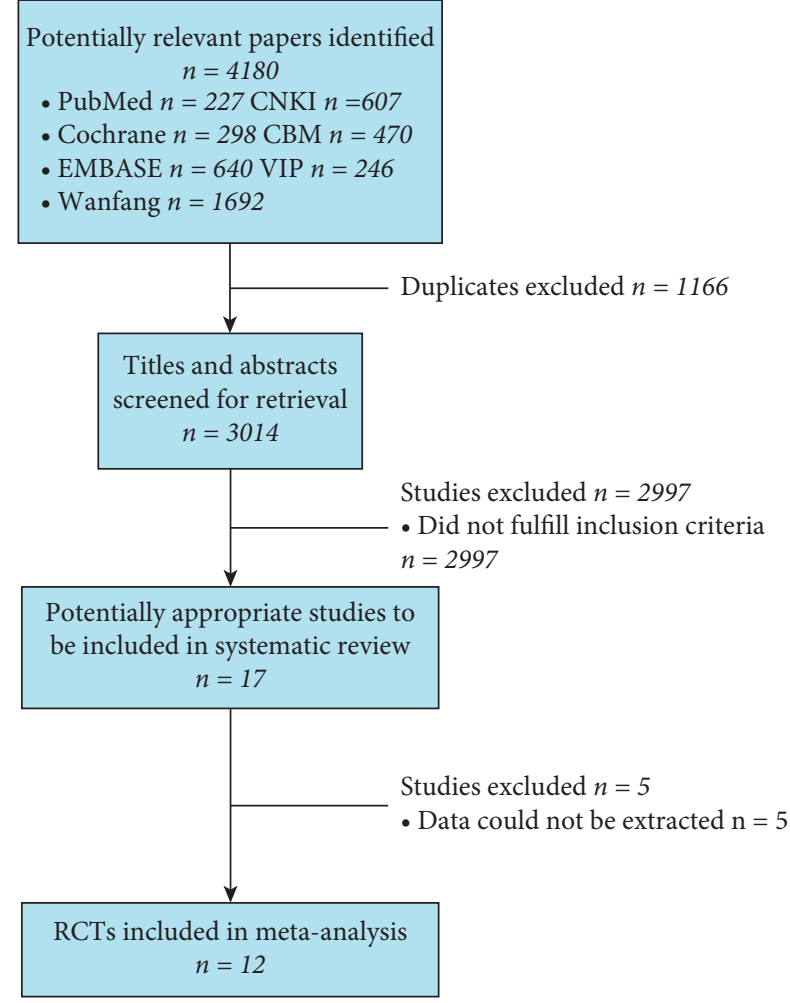

FIgURE 1: PRISMA flow diagram.
3.2. Study Characteristics. A total of 12 randomized controlled trials [25-36] were included in this study, with a total of 979 patients with UR (483 in the experimental group and 496 in the control group). All participants were Chinese. The intervention measure in the experimental group was electric acupuncture or manual acupuncture. In the control group, seven groups $[25,26,28,30-33]$ received drug intervention, of which six groups [25, 26, 30-33] received intramuscular injection of neostigmine, and one group [28] received oral Qianlieantong and finasteride tablets. The remaining patients in the control group were treated with conventional symptomatic treatment. All trials report on spontaneous urination, while three trials $[28,29,31]$ report changes in RUV. The characteristics of included studies are presented in Table 2.

3.3. Risk of Bias in Included Studies. The 12 papers included in this study were all randomized controlled trials. Four studies $[25,26,30,34]$ did not report the specific method of random generation, while 5 studies [27, 31-33, 35] used random methods with a higher risk of bias, such as the order of visits [31, 32], order of consultations [27], surgical duration [33], and time of disease onset [35]. None of the studies mentioned the application of allocation hiding related information. In terms of blinding, none of the studies described patient or evaluator blinding. All included randomized controlled trials had a low risk of bias against incomplete data and selective reporting. The risk of bias assessment is summarized in Figures 2 and 3. 


\subsection{Meta-Analysis Results}

3.4.1. Spontaneous Urination. All studies reported changes in the number of people with spontaneous urination after acupuncture. Statistics revealed heterogeneity among the 12 trials $\left(P<0.00001, I^{2}=80 \%\right)$. Therefore, a random-effects model was used to conduct a meta-analysis on the acupuncture group and the control group. The results show that acupuncture can effectively promote spontaneous urination and reduce anxiety in patients with poor urination (RR: 1.35; 95\% CI: $1.19-1.53 ; P<0.00001)$. The results of the metaanalysis are shown in Figure 4.

3.4.2. RUV. Three of the 12 studies reported changes in RUV after acupuncture. Statistics revealed heterogeneity among the three randomized controlled trials $\left(P<0.00001, I^{2}=99 \%\right)$. The random-effects model showed a significant difference in RUV between the acupuncture group and the control group (MD: $-84.79,95 \% \mathrm{CI}:-135.62$ to $-33.94 ; P=0.001)$. This indicates that acupuncture can effectively relieve UR. The results of this meta-analysis are shown in Figure 5.

3.4.3. Subgroup Analyses. We divided the included studies into three subgroups according to the interventions used in the experimental group, the interventions used in the control group, and the disease type to discuss the efficacy of acupuncture and study heterogeneity.

(1) Interventions in the Experimental Group. The acupuncture group was divided into electric acupuncture and manual acupuncture. Six articles used electric acupuncture, and six articles used manual acupuncture. Statistics showed significant heterogeneity in the treatment of UR with electrical acupuncture $\left(P=0.0003, I^{2}=76 \%\right)$, and there was a significant difference in the effective rate between the electrical acupuncture group and the control group ( $P=0.004$, RR: $1.22,95 \%$ CI: 1.06 to 1.40$)$. Moreover, there was a significant difference in the effective rate between the manual acupuncture group and the control group $(P<0.0001$, RR: $1.51,95 \%$ CI: 1.34 to 1.69$)$, but there was no significant heterogeneity $\left(P=0.43 P=0.43, I^{2}=0 \%\right)$. The results of this meta-analysis are shown in Figure 6.

(2) Interventions in the Control Group. Based on the interventions in the control group, this group was divided into a neostigmine group and a non-neostigmine group according to whether or not neostigmine was used. Seven of 12 articles used neostigmine in the control group, while the remaining 5 did not. Pooled data show a significant difference in the effective rate between the acupuncture group and the neostigmine group $(P<0.00001, \mathrm{RR}: 1.37,95 \% \mathrm{CI}$ : 1.19 to 1.57$)$ with obvious heterogeneity $\left(P=0.02, I^{2}=60 \%\right)$. A significant difference was observed in treatment effectiveness between the acupuncture group and the nonneostigmine control group $(P=0.01$, RR: $1.32,95 \%$ CI: 1.06 to 1.66), and marked heterogeneity was observed $\left(P<0.0001, I^{2}=87 \%\right)$. The results of this meta-analysis are shown in Figure 7.

3.5. Publication Bias. We conducted a funnel plot analysis of the 12 randomized controlled trials to analyze publication bias. The inverted funnel in Figure 8 is asymmetric on the left and right sides. A trim-and-fill analysis found that the results mostly fell in the shadow of $P<0.1$, indicating that there may be unpublished documents that are not statistically significant, which may lead to publication bias. In addition, since most of the included studies were in Chinese, language bias may be present.

\section{Discussion}

4.1. Principal Findings. This study shows that acupuncture has obvious advantages over conventional treatment in improving UR. Acupuncture can promote spontaneous urination to relieve the symptoms of UR and reduce RUV. In terms of safety, no studies mentioned adverse events, so the safety of acupuncture for UR could not be verified. Due to the high heterogeneity in this study, we divided the study into three subgroups according to the intervention used in the experimental group, the intervention used in the control group, and the disease type. The results show that heterogeneity was significantly reduced when the test group was classified by the type of acupuncture; thus, it can be inferred that the type of acupuncture may have been the source of the observed heterogeneity.

4.2. Mechanism of Acupuncture. UR usually occurs after childbirth, myelopathy, or lumbar spine surgery. There are many reasons for UR. First, damage to nerves that control the interaction between the bladder and the brain may cause loss of bladder control, leading to UR. Second, dysfunction in muscles or nerves that control urination may affect normal bladder and ureter function, leading to bladder dysfunction or failure of pelvic floor relaxation, resulting in UR [36]. Studies have shown that the direct nerve signal that induces bladder contraction may originate from the Barrington nucleus [37]. The Barrington nucleus is the core component of the urinary circuit. It increases the possibility of urination by activating the spinal excitatory pathway or inhibiting the spinal inhibitory mechanism [38]. In animal experiments, acupuncture changes the discharge characteristics of neurons related to bladder activity in and around the Barrington nucleus, thereby regulating bladder urination $[39,40]$.

According to traditional Chinese medicine, the kidney governs water balance, which means that metabolism of body fluids depends on the Qi transformation of kidney essence. The kidney has an exterior-to-interior relationship with the bladder, and normal transformationtransportation of kidney and bladder is responsible for urine production and excretion. An abnormal bladder Qi 


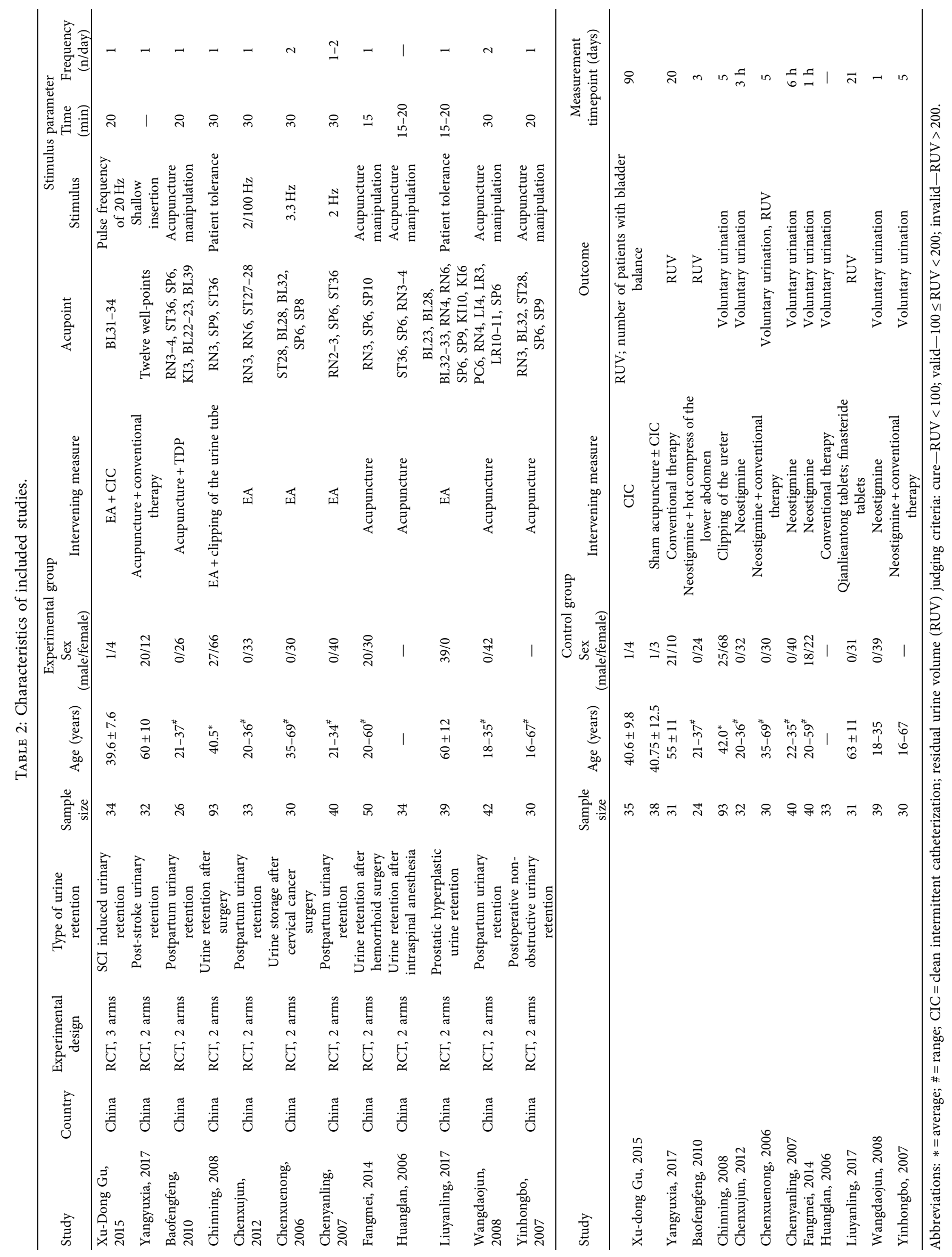


Random sequence generation (selection bias)

Allocation concealment (selection bias) Blinding of participants and personnel (performance bias) Blinding of outcome assessment (detection bias) Incomplete outcome data (attrition bias) Selective reporting (reporting bias) Other bias

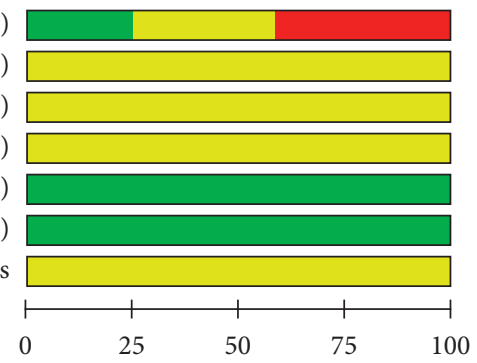

(\%)

Low risk of bias

$\square$ Unclear risk of bias

High risk of bias

FiguRe 2: Diagram of the risk of bias.

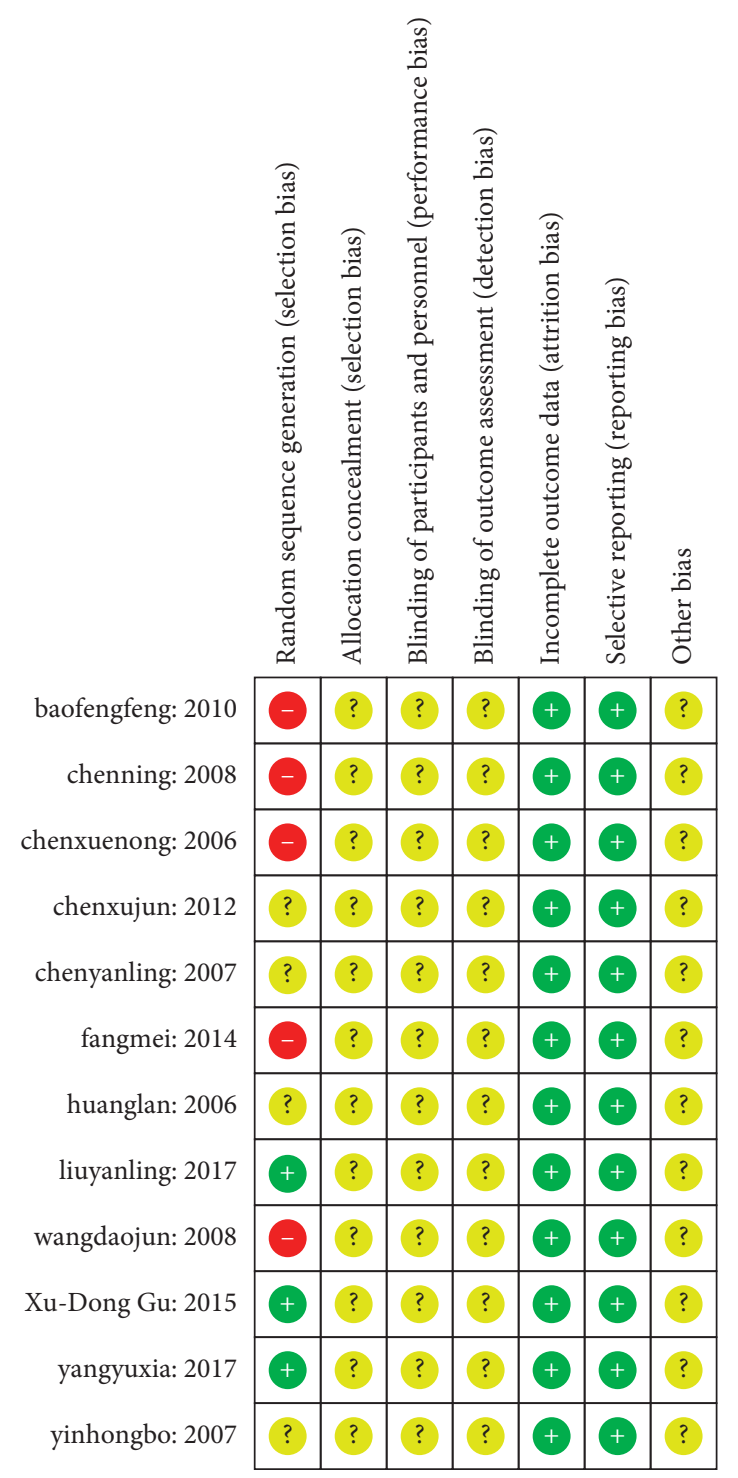

FIgURE 3: Summarized risk of bias. 


\begin{tabular}{|c|c|c|c|c|c|c|c|c|c|c|}
\hline \multirow{3}{*}{$\begin{array}{l}\text { Study or Subgroup } \\
\text { baofengfeng; } 2010\end{array}$} & \multicolumn{2}{|c|}{ Experimental } & \multicolumn{2}{|c|}{ Control } & \multirow{3}{*}{$\begin{array}{c}\text { Weight } \\
7.1 \%\end{array}$} & \multirow{3}{*}{$\begin{array}{c}\text { Risk Ratio } \\
\text { M-H, Random, 95\% CI }\end{array}$} & \multirow{2}{*}{\multicolumn{3}{|c|}{$\begin{array}{c}\text { Risk Ratio } \\
\text { M-H, Random, 95\% CI }\end{array}$}} & \\
\hline & \multirow{2}{*}{$\begin{array}{c}\text { Events } \\
24\end{array}$} & \multirow{2}{*}{$\frac{\text { Total }}{26}$} & \multirow{2}{*}{$\begin{array}{c}\text { Events } \\
17\end{array}$} & \multirow{2}{*}{$\begin{array}{c}\text { Total } \\
24\end{array}$} & & & & & & \\
\hline & & & & & & & & & & \\
\hline chenning; 2008 & 90 & 93 & 72 & 93 & $9.9 \%$ & $1.25[1.11,1.40]$ & & & & \\
\hline chenxuenong; 2006 & 29 & 30 & 21 & 30 & $7.7 \%$ & $1.38[1.08,1.76]$ & & & & \\
\hline chenxujun; 2012 & 31 & 33 & 23 & 32 & $7.9 \%$ & $1.31[1.03,1.65]$ & & & & \\
\hline chenyanling; 2007 & 39 & 40 & 35 & 40 & $9.7 \%$ & $1.11[0.98,1.27]$ & & & & \\
\hline fangmei; 2014 & 49 & 50 & 25 & 40 & $7.7 \%$ & $1.57[1.23,2.00]$ & & & & \\
\hline huanglan; 2006 & 32 & 34 & 16 & 33 & $5.8 \%$ & $1.94[1.35,2.79]$ & & & & \\
\hline liuyanling; 2017 & 37 & 39 & 30 & 31 & $10.1 \%$ & $0.98[0.89,1.08]$ & & & & \\
\hline wangdaojun; 2008 & 39 & 42 & 21 & 39 & $6.7 \%$ & $1.72[1.27,2.33]$ & & & & \\
\hline Xu-Dong Gu; 2015 & 15 & 17 & 21 & 35 & $6.4 \%$ & $1.47[1.07,2.03]$ & & & & \\
\hline Xu-Dong Gu; 2015 & 14 & 17 & 23 & 38 & $6.1 \%$ & $1.36[0.97,1.91]$ & & & & \\
\hline yangyuxia; 2017 & 29 & 32 & 21 & 31 & $7.3 \%$ & $1.34[1.02,1.75]$ & & & & \\
\hline yinhongbo; 2007 & 29 & 30 & 20 & 30 & $7.4 \%$ & $1.45[1.12,1.88]$ & & & & \\
\hline Total (95\% CI) & & 483 & & 496 & $100.0 \%$ & $1.35[1.19,1.53]$ & & & & \\
\hline Total events & 457 & & 345 & & & & & & & \\
\hline \multicolumn{7}{|c|}{ Heterogeneity: $\mathrm{Tau}^{2}=0.04 ; \mathrm{Chi}^{2}=59.46, \mathrm{df}=12(\mathrm{P}<0.00001) ; I^{2}=80 \%$} & 1 & 1.7 & $\begin{array}{l}1 \\
1.5\end{array}$ & $\begin{array}{l}1 \\
2\end{array}$ \\
\hline \multicolumn{7}{|c|}{ Test for overall effect: $Z=4.57(P<0.00001)$} & \multicolumn{4}{|c|}{ Favours [control] } \\
\hline
\end{tabular}

FIgURE 4: Results of the meta-analysis on spontaneous urination.

transformation can induce UR, resulting in a low urine volume, dripping out, or even occlusion. Simulating acupoints can unblock the meridians and regulate internal organs. According to basic theories of traditional Chinese medicine, acupuncture can promote Qi transformation of the kidney and bladder and restore bladder function. The effectiveness of acupuncture depends on techniques that are difficult for doctors to master. These techniques include needle insertion angle and depth, as well as the holding force before the needle is removed. Use of different techniques by different practitioners will impact the effects of treatment.

4.3. Implications for Clinical Practice and Further Research. This study shows that acupuncture is a safe and convenient treatment method that has a good effect on postpartum and postoperative UR. Frequently used acupuncture points include Ciliao (BL32), Sanyinjiao (SP6), Zhongji (RN3), and Guanyuan (RN4). Further research and screening of fixed and effective acupoints should be carried out to form a fixed acupuncture treatment plan to benefit more patients with UR. High-quality, large-scale, multi-center, clinical randomized controlled studies are required to obtain more accurate analysis results. Further research should adopt strict randomization, allocation concealment, and blinding, as well as clear inclusion and exclusion criteria, and the criteria for curing disease should be standardized and unified.

4.4. Advantages of This Research. The current systematic review focused on postpartum UR and drug intervention. No up-to-date systematic reviews on acupuncture interventions have been published in the last two years.
Moreover, most previous systematic reviews only studied a single type of UR or a single type of intervention in the control group. As far as we know, this study is the first systematic review and meta-analysis to comprehensively evaluate the effectiveness of acupuncture and moxibustion in the treatment of UR without limiting the type of UR or the type of intervention in the control group. In the present study, the effectiveness of acupuncture in the treatment of UR has been clarified to a certain extent. We mainly focused on the effects of common forms of acupuncture, which reduced the possible variability in other forms of acupuncture and moxibustion and enabled a more accurate assessment of the role of acupuncture in UR. We also screened the most frequently used acupoints from the included literature. We believe the findings of this study will aid future research on acupuncture in the treatment of UR.

4.5. Limitations. The limitations of this study are as follows. First, due to lack of high-quality, multi-center, clinical randomized controlled studies, the level of evidence in this study was limited. Second, publication bias exists, and the analysis results are not sufficiently robust. Third, only objective indicators, such as spontaneous urination and RUV, which are closely related to clinical practice, were evaluated. Conversely, indicators that are difficult to systematically divide and that are clinically heterogeneous, such as curative effect grade and patient experience, were not analyzed. Finally, due to lack of reports on the adverse effects of acupuncture, the possible adverse reactions during implementation of acupuncture were not systematically evaluated. 


\begin{tabular}{|c|c|c|c|c|c|c|c|c|c|c|c|c|}
\hline \multirow{3}{*}{$\begin{array}{l}\text { Study or Subgroup } \\
\text { liuyanling; } 2017\end{array}$} & \multicolumn{3}{|c|}{ Experimental } & \multicolumn{3}{|c|}{ Control } & \multirow{3}{*}{$\begin{array}{l}\text { Weight } \\
25.2 \%\end{array}$} & \multirow{3}{*}{$\begin{array}{c}\text { Mean Difference } \\
\text { IV, Random, 95\% CI }\end{array}$} & \multirow{2}{*}{\multicolumn{4}{|c|}{$\begin{array}{c}\text { Mean Difference } \\
\text { IV, Random, 95\% CI }\end{array}$}} \\
\hline & \multirow{2}{*}{$\frac{\text { Mean }}{-66.3}$} & \multirow{2}{*}{$\frac{\mathrm{SD}}{18.3}$} & \multirow{2}{*}{$\frac{\text { Total }}{39}$} & \multirow{2}{*}{$\frac{\text { Mean }}{-70.71}$} & \multirow{2}{*}{$\begin{array}{l}\mathrm{SD} \\
19.4\end{array}$} & \multirow{2}{*}{$\begin{array}{c}\text { Total } \\
31\end{array}$} & & & & & & \\
\hline & & & & & & & & & & - & 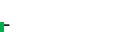 & \\
\hline Xu-Dong Gu; 2015 & -477.5 & 10.9 & 17 & -372.5 & 9.9 & 35 & $25.3 \%$ & $-105.00[-111.13,-98.87]$ & & - & & \\
\hline Xu-Dong Gu; 2015 & -477.5 & 10.9 & 17 & -398.5 & 9.9 & 38 & $25.3 \%$ & $-79.00[-85.06,-72.94]$ & & $=$ & & \\
\hline yangyuxia; 2017 & -229.8 & 37.8 & 32 & -67.65 & 46.9 & 31 & $24.3 \%$ & $-162.15[-183.22,-141.08]$ & -- & & & \\
\hline Total (95\% CI) & & & 105 & & & 135 & $100.0 \%$ & $-84.79[-135.62,-33.96]$ & & $\longrightarrow$ & & \\
\hline Heterogeneity: $\mathrm{Tau}^{2}$ & $=2652.18$ & $8 ; \mathrm{Chi}^{2}$ & ${ }^{2}=462$ & $2.92, \mathrm{df}=$ & $3(P<$ & $<0.000$ & $001) ; I^{2}=$ & $99 \%$ & -200 & -100 & 100 & 200 \\
\hline Test for overall effect & $Z=3.27$ & $7(P=$ & $0.001)$ & & & & & & Favours [e & experimental] & Favours [c & trol] \\
\hline
\end{tabular}

FIGURE 5: Meta-analysis results of RUV.

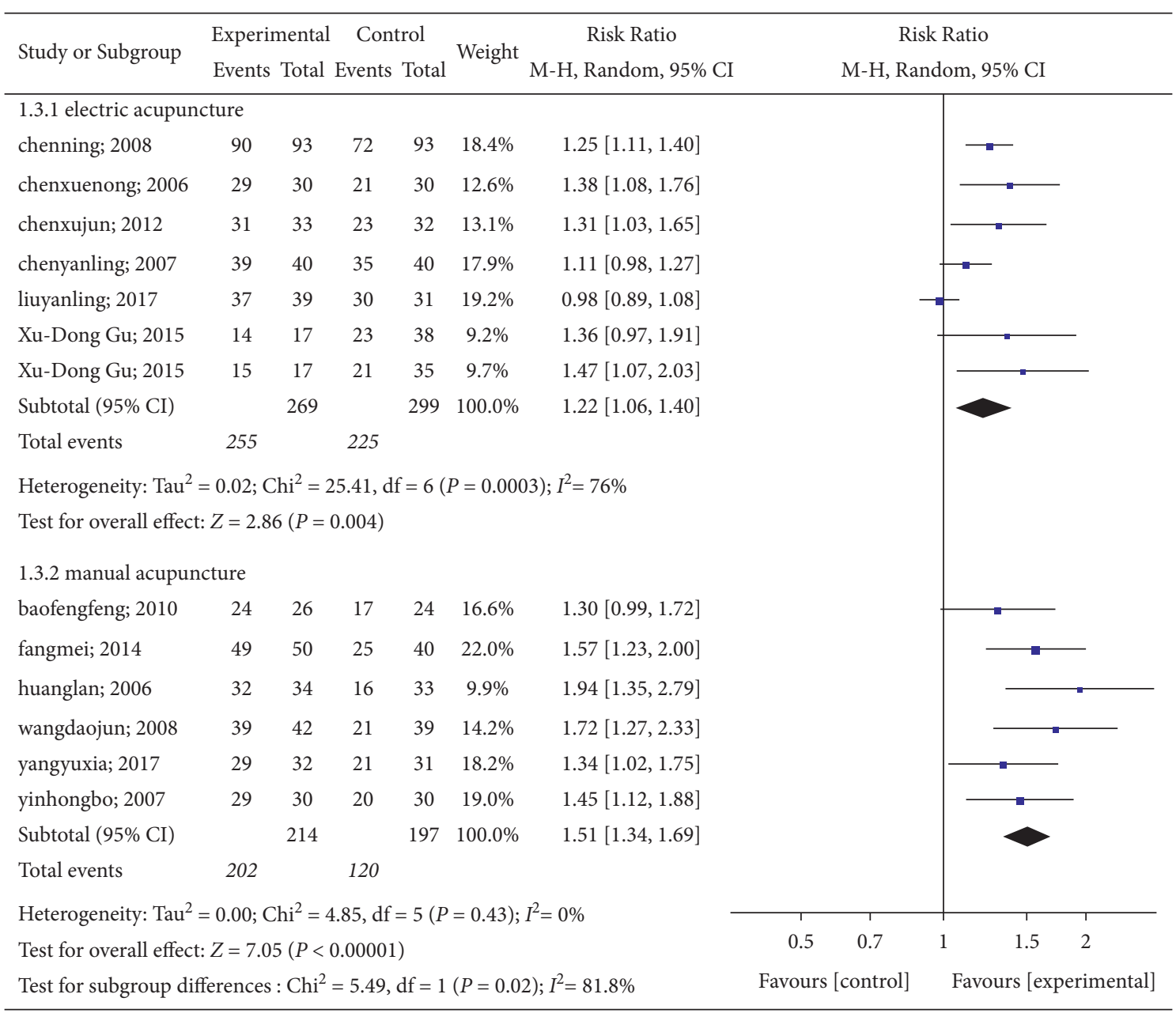

FIGURE 6: A meta-analysis of the effects of acupuncture type on spontaneous urination. 


\begin{tabular}{|c|c|c|c|c|c|c|c|c|c|}
\hline \multirow{2}{*}{$\begin{array}{l}\text { Study or Subgroup } \\
\text { 3.1.1 Neostigmine }\end{array}$} & \multicolumn{2}{|c|}{$\begin{array}{l}\text { Experimental } \\
\text { Events Total }\end{array}$} & \multicolumn{2}{|c|}{$\begin{array}{l}\text { Control } \\
\text { Events Total }\end{array}$} & Weight & $\begin{array}{c}\text { Risk Ratio } \\
\text { M-H, Random, 95\% CI }\end{array}$ & \multicolumn{3}{|c|}{$\begin{array}{c}\text { Risk Ratio } \\
\text { M-H, Random, 95\% CI }\end{array}$} \\
\hline & & & & & & & & & \\
\hline baofengfeng; 2010 & 24 & 26 & 17 & 24 & $12.3 \%$ & $1.30[0.99,1.72]$ & & & \\
\hline chenxuenong; 2006 & 29 & 30 & 21 & 30 & $14.0 \%$ & $1.38[1.08,1.76]$ & & & \\
\hline chenxujun; 2012 & 31 & 33 & 23 & 32 & $14.6 \%$ & $1.31[1.03,1.65]$ & & & \\
\hline chenyanling; 2007 & 39 & 40 & 35 & 40 & $20.5 \%$ & $1.11[0.98,1.27]$ & & $\rightarrow$ & \\
\hline fangmei; 2014 & 49 & 50 & 25 & 40 & $14.0 \%$ & $1.57[1.23,2.00]$ & & & \\
\hline wangdaojun; 2008 & 39 & 42 & 21 & 39 & $11.4 \%$ & $1.72[1.27,2.33]$ & & & \\
\hline yinhongbo; 2007 & 29 & 30 & 20 & 30 & $13.2 \%$ & $1.45[1.12,1.88]$ & & & \\
\hline Subtotal (95\% CI) & & 251 & & 235 & $100.0 \%$ & $1.37[1.19,1.57]$ & & & \\
\hline Total events & 240 & & 162 & & & & & & \\
\hline \multicolumn{10}{|c|}{ Heterogeneity: $\mathrm{Tau}^{2}=0.02 ; \mathrm{Chi}^{2}=15.11, \mathrm{df}=6(P=0.02) ; I^{2}=60 \%$} \\
\hline \multicolumn{10}{|c|}{ Test for overall effect: $Z=4.43(P<0.00001)$} \\
\hline \multicolumn{10}{|l|}{ 3.1.2 not neostigmine } \\
\hline chenning; 2008 & 90 & 93 & 72 & 93 & $20.2 \%$ & $1.25[1.11,1.40]$ & & 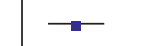 & \\
\hline huanglan; 2006 & 32 & 34 & 16 & 33 & $13.7 \%$ & $1.94[1.35,2.79]$ & & & \\
\hline liuyanling; 2017 & 37 & 39 & 30 & 31 & $20.5 \%$ & $0.98[0.89,1.08]$ & \multicolumn{3}{|c|}{$\rightarrow$} \\
\hline Xu-Dong Gu; 2015 & 14 & 17 & 21 & 35 & $14.1 \%$ & $1.37[0.97,1.95]$ & & & \\
\hline Xu-Dong Gu; 2015 & 15 & 17 & 23 & 38 & $15.1 \%$ & $1.46[1.07,1.99]$ & & 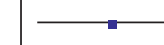 & \\
\hline yangyuxia; 2017 & 29 & 32 & 21 & 31 & $16.4 \%$ & $1.34[1.02,1.75]$ & & & \\
\hline Subtotal $(95 \% \mathrm{CI})$ & & 232 & & 261 & $100.0 \%$ & $1.32[1.06,1.66]$ & & & \\
\hline Total events & 217 & & 183 & & & & & & \\
\hline \multicolumn{10}{|c|}{ Heterogeneity: $\mathrm{Tau}^{2}=0.06 ; \mathrm{Chi}^{2}=38.35, \mathrm{df}=5(P<0.00001) ; I^{2}=87 \%$} \\
\hline \multicolumn{7}{|c|}{ Test for overall effect: $Z=2.47(P=0.01)$} & 0.7 & 1 & 2 \\
\hline \multicolumn{7}{|c|}{ Test for subgroup differences : $\mathrm{Chi}^{2}=0.06 \mathrm{df}=1(P<0.81) ; I^{2}=0 \%$} & \multicolumn{3}{|c|}{ Favours [experimental] } \\
\hline
\end{tabular}

FIgURE 7: A meta-analysis of the effects of neostigmine on spontaneous urination.

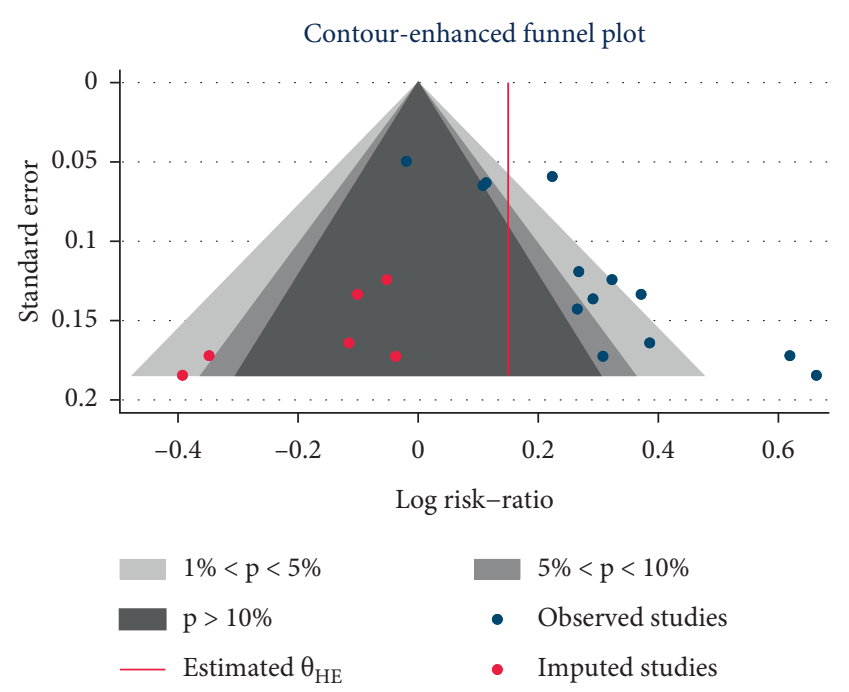

Figure 8: Publication bias and trim-and-fill analysis.

\section{Conclusion}

This meta-analysis of 12 randomized controlled trials shows that acupuncture is safe and effective in the treatment of UR.
However, since the current level of evidence is limited, highquality, large-sample, multi-center, clinical randomized controlled studies are needed to further confirm the conclusions in the future.

\section{Conflicts of Interest}

The authors have no conflicts of interest to declare.

\section{Authors' Contributions}

Chengwen Zheng and Zaoying Li contributed equally to this work.

\section{Acknowledgments}

The authors thank Zhao Kun of Zhejiang University for his guidance on this article.

\section{Supplementary Materials}

S1: checklist of items to include when reporting a systematic review or meta-analysis (PRISMA). (Supplementary Materials) 


\section{References}

[1] K. M. C. Verhamme, M. C. J. M. Sturkenboom, B. H. C. Stricker, and R. Bosch, "Drug-induced urinary retention,” Drug Safety, vol. 31, no. 5, pp. 373-388, 2008.

[2] J. T. Stoffel, "Non-neurogenic chronic urinary retention: what are we treating?" Current Urology Reports, vol. 18, no. 9, p. 74, 2017.

[3] S. A. Kaplan, A. J. Wein, D. R. Staskin, C. G. Roehrborn, and W. D. Steers, "Urinary retention and post-void residual urine in men: separating truth from tradition," The Journal of Urology, vol. 180, no. 1, pp. 47-54, 2008.

[4] D. M. Darrah, T. L. Griebling, and J. H. Silverstein, "Postoperative urinary retention," Anesthesiology Clinics, vol. 27, no. 3, pp. 465-484, 2009.

[5] G. Baldini, H. Bagry, A. Aprikian, F. Carli, D. S. Warner, and M. A. Warner, "Postoperative urinary retention," Anesthesiology, vol. 110, no. 5, pp. 1139-1157, 2009.

[6] R. Hamid, M. A. Averbeck, H. Chiang et al., "Epidemiology and pathophysiology of neurogenic bladder after spinal cord injury," World Journal of Urology, vol. 36, no. 10, pp. 1517-1527, 2018.

[7] H.-K. Le, D. Cardona-Grau, and G. Chiang, "Evaluation and long-term management of neurogenic bladder in spinal dysraphism," NeoReviews, vol. 20, no. 12, pp. e711-e724, 2019.

[8] L. W. Chan, T. L. Griebling, E. P. Arnold, P. S. Chu, P. W. New, and A. Wagg, "Special considerations in the urological management of the older spinal cord injury patient," World Journal of Urology, vol. 36, no. 10, pp. 1603-1611, 2018.

[9] W. Al Taweel and R. Seyam, "Neurogenic bladder in spinal cord injury patients," Research and Reports in Urology, vol. 7, pp. 85-99, 2015.

[10] R. J. Hurlbert, M. N. Hadley, B. C. Walters et al., "Pharmacological therapy for acute spinal cord injury," Neurosurgery, vol. 72, no. suppl_3, pp. 93-105, 2013.

[11] N. Sekido, Y. Igawa, H. Kakizaki et al., "Clinical guidelines for the diagnosis and treatment of lower urinary tract dysfunction in patients with spinal cord injury," International Journal of Urology, vol. 27, no. 4, pp. 276-288, 2020.

[12] D. G. Tate, T. Wheeler, G. I. Lane et al., "Recommendations for evaluation of neurogenic bladder and bowel dysfunction after spinal cord injury and/or disease," The Journal of Spinal Cord Medicine, vol. 43, no. 2, pp. 141-164, 2020.

[13] E. Kreydin, H. Zhong, K. Latack, S. Ye, V. R. Edgerton, and P. Gad, "Transcutaneous electrical spinal cord neuromodulator (TESCoN) improves symptoms of overactive bladder," Frontiers in Systems Neuroscience, vol. 14, p. 1, 2020.

[14] G. Markopoulos, D. Kitridis, K. Tsikopoulos, D. Georgiannos, and I. Bisbinas, "Bladder training prior to urinary catheter removal in total joint arthroplasty. A randomized controlled trial," International Journal of Nursing Studies, vol. 89, pp. 14-17, 2019.

[15] K. Schumm and T. B. L. Lam, "Types of urethral catheters for management of short-term voiding problems in hospitalized adults: a short version Cochrane review," Neurourology and Urodynamics, vol. 27, no. 8, pp. 738-746, 2008.

[16] B. S. Buckley and M. C. M. Lapitan, "Drugs for treatment of urinary retention after surgery in adults," Cochrane Database of Systematic Reviews, vol. 10, p. CD008023, 2010.

[17] M. D. Seidman, R. K. Gurgel, S. Y. Lin et al., "Clinical practice guideline," Otolaryngology-Head and Neck Surgery, vol. 152, no. 1_suppl, pp. S1-S43, 2015.
[18] J.-J. Zhang, J. Wang, P. Bai, C. Tan, Z.-C. Wang, and S. Chen, "Evidence-based clinical practice guideline of acupuncture and moxibustion for primary dysmenorrhea: development and characteristics," Zhongguo Zhen Jiu, vol. 39, no. 12, pp. 1343-1346, 2019.

[19] L. L. D. Zhong, W. Kun, N. Shi et al., "Evidence-based Chinese medicine clinical practice guideline for stroke in Hong Kong," Chinese Medicine, vol. 15, no. 1, p. 116, 2020.

[20] H. V. Acar, "Acupuncture and related techniques during perioperative period: a literature review," Complementary Therapies in Medicine, vol. 29, pp. 48-55, 2016.

[21] J. C. Forde, E. Jaffe, B. V. Stone, A. E. Te, G. Espinosa, and B. Chughtai, "The role of acupuncture in managing overactive bladder; a review of the literature," International Urogynecology Journal, vol. 27, no. 11, pp. 1645-1651, 2016.

[22] M. Oelke, A. Bachmann, A. Descazeaud et al., "EAU guidelines on the treatment and follow-up of non-neurogenic male lower urinary tract symptoms including benign prostatic obstruction," European Urology, vol. 64, no. 1, pp. 118-140, 2013.

[23] K. T. McVary, C. G. Roehrborn, A. L. Avins et al., "Update on AUA guideline on the management of benign prostatic hyperplasia," The Journal of Urology, vol. 185, no. 5, pp. 1793-1803, 2011.

[24] X. D. Gu, J. Wang, P. Yu et al., "Effects of electroacupuncture combined with clean intermittent catheterization on urinary retention after spinal cord injury: a single blind randomized controlled clinical trial," International Journal of Clinical and Experimental Medicine, vol. 8, no. 10, pp. 19757-19763, 2015.

[25] X.-J. Chen and W. Zheng, "Treatment of 33 cases of postpartum urinary retention with dense wave needle," Guangming Journal of Chinese Medicine, vol. 27, no. 1, pp. 92-93, 2012.

[26] Y.-L. Chen, "Forty cases of postpartum urinary retention treated by electroacupuncture," Shanghai Journal of Acu-Mox, vol. 26, no. 1, 2007.

[27] X.-N. Chen, Z.-Z. Zhang, and K.-Z. Zhao, "Observations on therapeutic effect of electroacupuncture on 60 cases of urinary retention after cervical cancer operation," Shanghai Journal of Acu-Mox, vol. 25, no. 03, pp. 13-14, 2006.

[28] Y.-L. Liu, X.-D. Wang, and S.-F. Huang, "Observations on therapeutic effect of electroacupuncture on urinary retention due to prostatic hyperplasia," Shanghai Journal of Acu-Mox, vol. 36, no. 11, pp. 1318-1320, 2017.

[29] Y. Yang, R. Xiang, Y. Liu, E. Chang, and Y. Huo, "Clinical study on post-stroke urinary retention treated with acupuncture at the twelve jing-well points and bladder function training," Chinese Acupuncture \& Moxibustion, vol. 37, no. 10, pp. 1041-1044, 2017.

[30] H.-B. Yin and S.-C. Wang, "Observations on therapeutic effect of acupuncture therapy on 60 cases of non-obstructive urinary retention after operation," Shandong Medical Journal, vol. 47, no. 26, p. 106, 2007.

[31] F.-F. Bao and A.-J. Yuan, "Acupuncture treatment of 26 cases of postpartum urinary retention," Jiangxi Journal of Traditional Chinese Medicine, vol. 41, no. 8, p. 55, 2010.

[32] D.-J. Wang, "Acupuncture treatment of 42 cases of refractory postpartum urinary retention," Journal of Anhui Traditional Chinese Medical College, vol. 27, no. 4, pp. 22-23, 2008.

[33] M. Fang and X.-Y. Hu, "Observations on therapeutic effect of acupuncture on 50 cases of urinary retention due to hemorrhoid surgery," Nei Mongol Journal of Traditional Chinese Medicine, vol. 33, no. 6, pp. 83-84, 2014. 
[34] L. Huang, C.-Y. Yang, and X. Yang, "Observations on therapeutic effect of acupuncture on relieving urinary retention after spinal anesthesia," The Western Journal of Medicine China, vol. 18, no. 5, p. 636, 2006.

[35] N. Chen and X.-W. Wang, "Observations on therapeutic effect of acupuncture on 186 cases of urinary retention after operation," China Practical Medical, vol. 3, no. 30, pp. 162-163, 2008.

[36] S. Chen, H. Sun, H. Xu, Y. Zhang, and H. Wang, "Effects of acupuncture on hospitalized patients with urinary retention," Evidence-based Complementary and Alternative Medicine, vol. 2020, Article ID 2520483, 7 pages, 2020.

[37] Y. Tanaka, Y. Koyama, Y. Kayama, A. Kawauchi, O. Ukimura, and T. Miki, "Firing of micturition center neurons in the rat mesopontine tegmentum during urinary bladder contraction," Brain Research, vol. 965, no. 1-2, pp. 146-154, 2003.

[38] M. Sasaki, "Role of Barrington's nucleus in micturition," The Journal of Comparative Neurology, vol. 493, no. 1, pp. 21-26, 2005.

[39] Y. Tanaka, Y. Koyama, E. Jodo et al., "Effects of acupuncture to the sacral segment on the bladder activity and electroencephalogram," Psychiatry and Clinical Neurosciences, vol. 56, no. 3, pp. 249-250, 2002.

[40] H. Wang, Y. Tanaka, A. Kawauchi, T. Miki, Y. Kayama, and Y. Koyama, "Acupuncture of the sacral vertebrae suppresses bladder activity and bladder activity-related neurons in the brainstem micturition center," Neuroscience Research, vol. 72, no. 1, pp. 43-49, 2012. 